\title{
On the application of the effective action approach to amplitudes with reggeon splitting
}

\author{
M. A. Braun, S. S. Pozdnyakov, M. Yu. Salykin, M. I. Vyazovsky ${ }^{\mathrm{a}}$ \\ Saint-Petersburg State University, St. Petersburg, Russia
}

Received: 20 February 2014 / Accepted: 19 July 2014 / Published online: 7 August 2014

(C) The Author(s) 2014. This article is published with open access at Springerlink.com

\begin{abstract}
Application of the effective action approach to amplitudes with loop integration is studied for collisions on two and three centers with possible gluon emission. A rule is formulated for the integration around pole singularities in the induced vertices which brings the results in agreement with the QCD. It is demonstrated that the amplitudes can be restored from the purely transverse picture by introducing the standard Feynman propagators for intermediate gluons and quarks.
\end{abstract}

\section{Introduction}

In the Regge kinematics, relevant for high-energy hadronic processes, in the framework of the perturbative QCD, the amplitudes can be conveniently constructed using the effective action proposed by Lipatov [1,2]. In this formalism gluons at fixed rapidities are described by the standard field $V_{\mu}=-i t^{a} V_{\mu}^{a}$. Regions with essentially different rapidities are connected by the reggeon field $A_{\mu}^{y}=-i t^{a} A_{\mu}^{y a}$ with only non-zero longitudinal components $A_{+}$and $A_{-}$, describing the reggeized gluons.

The effective Lagrangian is local in rapidity and describes the self-interaction of gluons at a given rapidity by means of the usual QCD Lagrangian $\mathcal{L}_{\mathrm{QCD}}$ and their interaction with reggeons. It has the form $[1,2]$

$$
\begin{aligned}
\mathcal{L}_{\mathrm{eff}}= & \mathcal{L}_{\mathrm{QCD}}\left(V_{\mu}^{y}+A_{\mu}^{y}\right)+2 \operatorname{Tr}\left(\left(\mathcal{A}_{+}\left(V_{+}^{y}+A_{+}^{y}\right)-A_{+}^{y}\right)\right. \\
& \left.\times \partial_{\perp}^{2} A_{-}^{y}+\left(\mathcal{A}_{-}\left(V_{-}^{y}+A_{-}^{y}\right)-A_{-}^{y}\right) \partial_{\perp}^{2} A_{+}^{y}\right),
\end{aligned}
$$

\footnotetext{
a e-mail: ovsky@mv8660.spb.edu
}

where

$$
\begin{aligned}
\mathcal{A}_{ \pm}\left(V_{ \pm}\right)= & \sum_{n=0}^{\infty}(-g)^{n} V_{ \pm}\left(\partial_{ \pm}^{-1} V_{ \pm}\right)^{n} \\
= & V_{ \pm}-g V_{ \pm} \partial_{ \pm}^{-1} V_{ \pm}+g^{2} V_{ \pm} \partial_{ \pm}^{-1} V_{ \pm} \partial_{ \pm}^{-1} V_{ \pm} \\
& +-\ldots
\end{aligned}
$$

The shift $V_{\mu} \rightarrow V_{\mu}+A_{\mu}$ with $A_{\perp}=0$ is done to exclude direct gluon-reggeon transitions. The reggeon fields are assumed to be subject to kinematical conditions $\partial_{-} A_{+}=\partial_{+} A_{-}=0$ and their propagator is in the momentum representation

$$
\left\langle A_{+}^{y a} A_{-}^{y^{\prime} b}\right\rangle=-i \frac{\delta_{a b}}{q_{\perp}^{2}} \theta\left(y^{\prime}-y\right) .
$$

Inspection of (2) shows that the new vertices generated by the effective action ('induced' vertices) contain poles at $\partial_{ \pm}=0$, which in the momentum representation correspond to vanishing of the longitudinal momenta transferred to the target or projectile. In fact these vertices can only be introduced when these momenta are different from zero. Otherwise the conditions of the reggeon kinematics are violated and the effective action cannot be applied. Thus effective action serves only to find induced vertices at non-zero values of the transferred longitudinal momenta. However, in the physical amplitudes these vertices are only a part of the whole contribution. They are to be connected with the projectile(s) and target(s) with reggeon propagators and in many cases integrated over the transferred longitudinal momenta. At this moment the problem of interpreting the mentioned singularities at zero values of these momenta arises.

Hermiticity of the effective Lagrangian suggests that from the start the singularities at $\partial_{ \pm}=0$ should possibly be interpreted in the Cauchy principal value prescription in the momentum representation. In our papers $[3,4]$ it was shown that for the scattering on two centers with gluon emission this prescription indeed produces correct amplitudes, which in the lowest order reproduce the standard QCD amplitudes. However, in a later paper M.Hentschinski discovered that for 
simple elastic scattering on three centers the principal value prescription for the effective Lagrangian violates the desired properties of the transition vertex of a reggeon into three reggeons (' $\mathrm{R} \rightarrow \mathrm{RRR}$ vertex') and in all probability to more reggeons $[5,6]$. To restore these properties M.Hentschinski proposed a recipe, which essentially consists in projecting the contribution of the vertex onto maximally antisymmetric color states in the crossed channel. Obviously this recipe is external to the effective action approach and should be invoked as an additional requirement. However, this recipe refers only to the vertex itself and does not cover the case when the vertices are inserted into the amplitudes as a whole and when it does not solve the problem.

In this paper we study this question in a more general framework and propose a different prescription. In the effective action the propagators of the projectile coupled to the reggeons should not be taken as the standard Feynman ones. Only the $\delta$-functional part of them should be retained in accordance with the fact that the effective action is local in rapidity [4].

Note that the product of dropped parts with principal value singularities may itself contain $\delta$-functional terms. So the recipe in [4] cannot be formulated as discarding all $\delta$ functional terms in the product of intermediate projectile propagators as a whole. Rather such terms should be dropped in each intermediate propagator.

We advocate that in accordance with the Regge kinematics one should operate with the induced vertex as if the transferred longitudinal momenta were different from zero. We show that after its transformation into a certain adequate form one can impose the prescription of principal value for the singularities of the effective action in the longitudinal momenta. The 'adequate form' is such that all the resulting propagators have the standard Feynman singularities.

Note that taking the transferred longitudinal momenta different from zero from the start we do not pretend to specify a description to circumvent the singularities. The question of the correct pole prescription is avoided at this point and in fact postponed for later analysis of the amplitude as a whole. In this analysis one indeed finds ambiguities as to discarding the $\delta$-functional terms. They are resolved in a unique manner by requiring that in the end one finds only the Feynman singularities as dictated by comparison with the QCD diagrams.

We shall demonstrate that this rule allows to obtain correct amplitudes from the effective action without any additional non-physical terms. One can mnemonically term this prescription by the rule that the induced vertices cannot contain any $\delta$-functions in the transferred longitudinal momenta, although the exact meaning of this rule is as presented above.

We also show that there exists an alternative way for the construction of amplitudes in the Regge kinematics, which completely avoids the mentioned singularity problem. Since many years ago, starting from [7-9] and followed by [10], it was shown that their multiple discontinuities can be constructed in a purely transverse picture containing certain effective vertices (Lipatov and Bartels vertices) for real gluon emission. We demonstrate that the amplitudes themselves can be restored from this transverse picture by connecting the particles (gluon and quarks) by standard Feyman propagators. In some cases this method proves to be simpler that the direct application of the effective action (see e.g. [11]).

The paper is organized as follows. In Sects. 2 and 3 we consider scattering on two centers without (Sect. 2) and with (Sect. 3) gluon emission. In this case the principal value prescription works. A part of the material in these sections has been already published in $[4,6]$ and is included to have a general view on the problem. The new results refer to the description in terms of Lipatov and Bartels vertices connected by Feynman propagators. In Sects. 4 and 5 we study scattering on three centers without (Sect. 4) and with (Sect. 5) gluon emission. Again a part of the material reproduces results of [12] necessary for the presentation but most of it is new.

In our study we simplify the projectile and targets to be quarks of momentum $k$ for the projectile and $l$ for each of the two or three targets. We assume $k_{-}=l_{+}=k_{\perp}=l_{\perp}=0$ and work in the c.m. system $k_{+}=l_{-}$. The color indices of reggeons attached to the targets and that of the emitted gluon are denoted as $b_{1}, b_{2}, b_{3}$, and $a$, respectively. To economize on notations we denote the products of projectile quark color matrices $t^{b_{1}} t^{b_{2}} t^{b_{3}}$ simply as (123) and their trace as [123]. Also the longitudinal momenta $q_{1-}, q_{2-}$, and $q_{3-}$ will be denoted as 1, 2, and 3 when this does not lead to confusion. Pole at zero values of longitudinal momenta will always be understood in the principal value sense, which will not be indicated explicitly. In our figures the normal gluons will be shown by solid lines and reggeons by wavy lines. The quark projectile will be shown by thick solid line. The induced vertices will be denoted by open circles with cross, the effective vertices of Lipatov and Bartels by simple and double dots.

\section{Elastic scattering off two centers}

\subsection{Lowest order QCD}

In QCD in the lowest order the amplitude for the elastic scattering off two centers in the axial gauge $(V l)=0$ is trivial: it is just the double gluon exchange, Fig. $1 A$, since the contribution of the diagram with the 3-gluon vertex Fig. $1 B$ is zero.

So the amplitude is

$$
A=16(k l)^{2} \frac{(21)}{\left(k-q_{1}\right)^{2}+i 0}+P_{12} .
$$

Here, as mentioned in the Introduction, $(21)=t^{b_{2}} t^{b_{1}} ; q_{1}$ is the momentum transferred to the right target. In the high- 


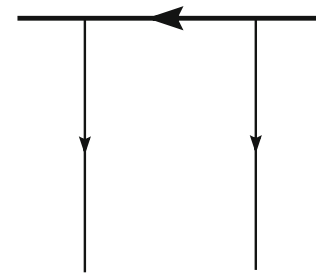

A

Fig. 1 Elastic scattering on two centers in the lowest order of the QCD. Here and in the following figures coupling of the reggeons to the target quarks is not explicitly shown

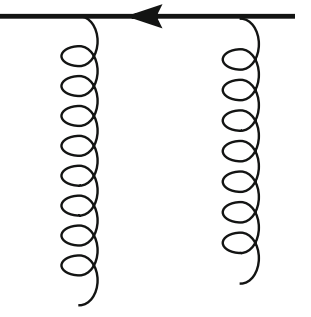

A

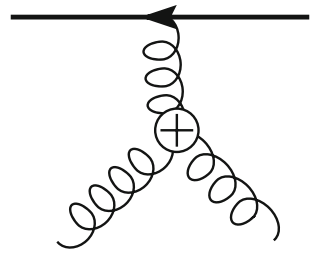

$B$
Fig. 2 Elastic scattering on two centers in the effective action approach

energy limit $q_{1+} \rightarrow 0$. Symbol $P_{12}$ means interchange $1 \leftrightarrow 2$. From the mass-conditions for the projectile it follows that $q_{1-}+q_{2-} \rightarrow 0$. The Regge kinematics assumes $\left|2 k_{+} q_{1-}\right|>>\left|q_{1 \perp}^{2}\right|$. Then $\left(k-q_{1}\right)^{2}=-2 k_{+} q_{1-}+q_{1 \perp}^{2} \simeq$ $-2 k_{+} q_{1-}$. In this limit the amplitude can be split into the principal value part and $\delta$-functional part as follows:

$$
\begin{aligned}
A= & 16(k l)^{2}\left[-i f^{b_{1} b_{2} c} t^{c} \mathrm{P} \frac{1}{2 k_{+} q_{1-}}\right. \\
& \left.-\pi \delta\left(2 k_{+} q_{1-}\right)\left\{t^{b_{1}} t^{b_{2}}\right\}\right]+P_{12} .
\end{aligned}
$$

\subsection{Effective action result}

In the effective action approach one has to retain only the $\delta$ functional part of the diagram in Fig. $2 \mathrm{~A}$ but take into account the diagram in Fig. $2 B$. Obviously to have the correct amplitude the latter has to reproduce the term with the principal value in (5).

The standard 3-gluon vertex, as before, gives zero in the axial gauge and only the induced vertex remains. This reggeon $\rightarrow$ two-reggeons vertex is given by

$\Gamma_{R \rightarrow R R}=\frac{\left(q_{1}+q_{2}\right)_{\perp}^{2}}{q_{1-}} f^{b_{1} b_{2} c}$.

Inserting it into the amplitude in Fig. $2 B$ we obtain the contribution

$$
A_{1}=-i 16(k l)^{2} f^{b_{1} b_{2} c} t^{c} \frac{1}{2 k_{+} q_{1-}} .
$$

Comparison with (5) demonstrates that if one understands the pole at $q_{1-}=0$ in (7) in the principal value sense then the effective action exactly reproduces the QCD result.

Note that from this result it follows that one can describe the scattering just by taking only diagrams A with the double reggeon exchange. In this way one avoids poles at $q_{1-}=0$ altogether and remains with the standard Feynman denominators. This presets a simple example of the alternative description of high-energy amplitudes in the Regge kinematics.

\section{Gluon emission off two centers}

\subsection{Lowest order QCD}

In the QCD at the lowest order it is described by 6 diagrams shown in Fig. $3 A-F$.

These diagrams in the Regge kinematics were calculated in [4]. It was found

$$
\begin{aligned}
A+B+C= & -32 i(k l)^{2} \frac{(e p)_{\perp}}{p_{\perp}^{2}}\left[\frac{f^{b_{1} a c}(2 c)}{\left(k^{\prime}+q_{2}\right)^{2}+i 0}\right. \\
& \left.-\frac{f^{a b_{2} c}(c 1)}{\left(k-q_{1}\right)^{2}+i 0}\right],
\end{aligned}
$$

$D=-32 i(k l)^{2}\left(e, p+q_{1}\right)_{\perp} \frac{f^{a b_{1} c}(2 c)}{\left[\left(k^{\prime}+q_{2}\right)^{2}+i 0\right]\left[\left(p+q_{1}\right)^{2}+i 0\right]}$,

$$
E=-32 i(k l)^{2}\left(e, p+q_{2}\right) \perp \frac{f^{a b_{2} c}(c 1)}{\left[\left(k-q_{1}\right)^{2}+i 0\right]\left[\left(p+q_{2}\right)^{2}+i 0\right]},
$$

$F=32(k l)^{2} \frac{p_{+}}{k_{+}} \frac{\left(e, p+q_{1}+q_{2}\right)_{\perp}}{\left(p+q_{1}+q_{2}\right)_{\perp}^{2}} \frac{f^{a b_{2} c} f^{c b_{1} d} t^{d}}{\left(p+q_{2}\right)^{2}+i 0}$.

Here $e, p$, and $a$ are the polarization vector, momentum, and color index of the emitted gluon; $k^{\prime}=k-q_{1}-q_{2}-p ;(2 c)=$ $t^{b_{2}} t^{c}$. To these diagrams also the ones with the interchange $1 \leftrightarrow 2$ should be added.

\subsection{Effective action results}

In the effective approach the same amplitude is described by only four diagrams shown in Fig. 4A-D.

In diagrams $A$ and $B$ the gluon is emitted by the Lipatov vertex. We have

$L\left(p, q_{1}\right)=\frac{(p e)_{\perp}}{p_{\perp}^{2}}-\frac{\left(p+q_{1}, e\right)_{\perp}}{\left(p+q_{1}\right)_{\perp}^{2}}$.

According to the rules of the effective action approach in these diagrams in the quark propagator only the $\delta$-functional term is to be retained. 
Fig. 3 Gluon emission on two centers in the lowest order of the QCD

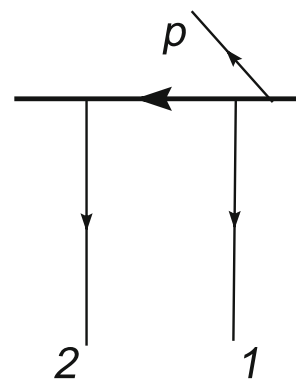

A

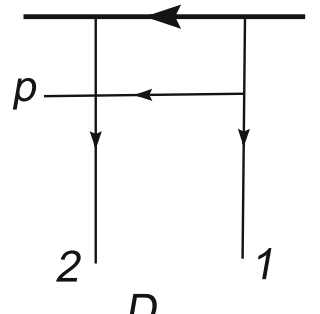

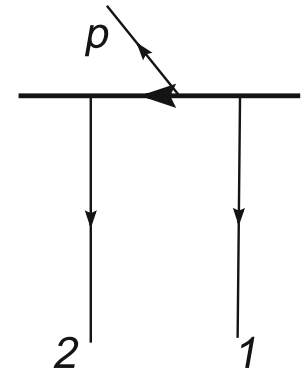

$B$

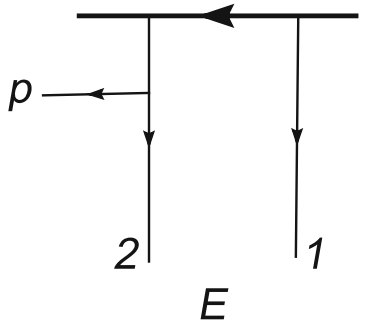

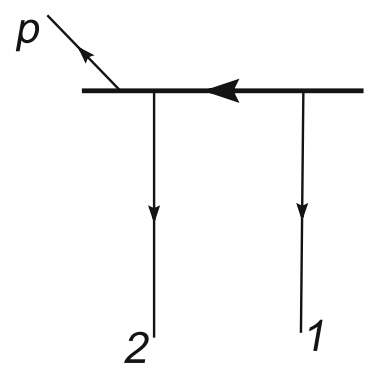

C

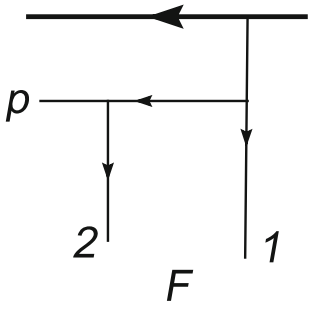

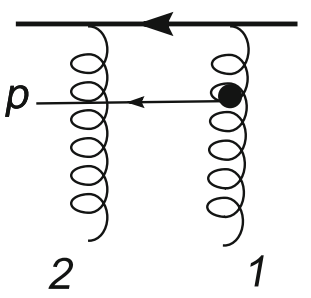

A

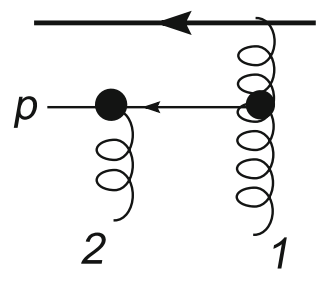

C

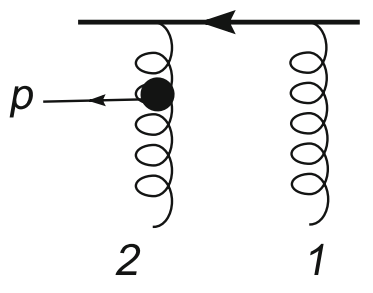

$B$

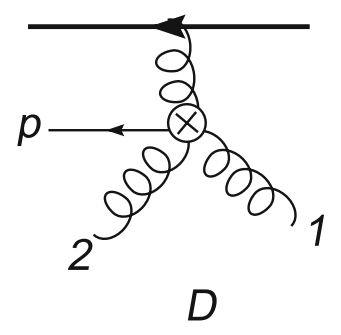

Fig. 4 Gluon emission on two centers in the effective action approach

The $R \rightarrow R R P$ vertex ( $P$ for "particle') which enters diagrams $C$ and $D$ was calculated in [3]. It can be split into the proper $(\mathrm{W})$ and induced $(\mathrm{R})$ parts,

$$
\begin{aligned}
& \Gamma_{R \rightarrow R R P}=W_{R \rightarrow R R P}+R_{R \rightarrow R R P} \\
& W_{R \rightarrow R R P}=-i \frac{2 q_{+} q_{\perp}^{2}}{\left(q_{2}+p\right)^{2}+i 0} f^{d b_{1} c} f^{c b_{2} a} B\left(p, q_{2}, q_{1}\right) \\
& R_{R \rightarrow R R P}=i \frac{q_{\perp}^{2}}{q_{1-}} f^{d b_{1} c} f^{c b_{2} a} L\left(p, q_{2}\right) .
\end{aligned}
$$

Here $B\left(p, q_{2}, q_{1}\right)=L\left(p+q_{2}, q_{1}\right)$ is the so-called Bartels vertex.

Inserted into the amplitude the proper part $\mathrm{W}$ does not give any trouble after integrations. However, in the induced part $\mathrm{R}$ we again meet with the pole at $q_{1-}=0$. In [4] it was demonstrated that summed with the $\delta$-functional term from the quark propagator the contribution from the $R \rightarrow R R P$ vertex contained in the part R exactly reproduces the $Q C D$ result provided one interprets the pole in (15) as the principal value. The necessity to remove $\delta$-functional terms from the induced vertex does not arise.

However, this is not so for scattering on three (and possible more) centers.

\subsection{Alternative form of the amplitude with gluon emission}

Here we present a different form of the amplitude in terms of the Lipatov and Bartels vertices, which does not involve poles at $q_{1,2-}=0$ and so does not require any additional information about these poles.

In the Regge kinematics

$$
\begin{aligned}
\frac{1}{\left(p+q_{1}\right)^{2}+i 0} & =\frac{1}{-2 p_{+} q_{2-}+\left(p+q_{1}\right)_{\perp}^{2}+i 0} \\
& =\frac{1}{\left(p+q_{1}\right)_{\perp}^{2}}\left(1+\frac{2 p_{+} q_{2-}}{\left(p+q_{1}\right)^{2}+i 0}\right) .
\end{aligned}
$$

Correspondingly we split the contribution $D$, see (10), to the amplitude in two parts: $D=D_{1}+D_{2}$, where

$D_{1}=-32 i(k l)^{2} \frac{\left(e, p+q_{1}\right)_{\perp}}{\left(p+q_{1}\right)_{\perp}^{2}} \frac{f^{a b_{1} c} t^{b_{2}} t^{c}}{\left(k^{\prime}+q_{2}\right)^{2}+i 0}$, 


$$
\begin{aligned}
D_{2}= & -32 i(k l)^{2} \frac{\left(e, p+q_{1}\right)_{\perp}}{\left(p+q_{1}\right)_{\perp}^{2}} f^{a b_{1} c} t^{b_{2}} t^{c} \\
& \times \frac{2 p_{+} q_{2}}{\left[\left(k^{\prime}+q_{2}\right)^{2}+i 0\right]\left[\left(p+q_{1}\right)^{2}+i 0\right]} .
\end{aligned}
$$

Similarly we split the contribution $E$, see (11), in two parts

$$
\begin{aligned}
E_{1}= & -32 i(k l)^{2} \frac{\left(e, p+q_{2}\right)_{\perp}}{\left(p+q_{2}\right)_{\perp}^{2}} \frac{f^{a b_{2} c} t^{c} t^{b_{1}}}{\left(k-q_{1}\right)^{2}+i 0}, \\
E_{2}= & -32 i(k l)^{2} \frac{\left(e, p+q_{2}\right)_{\perp}}{\left(p+q_{2}\right)_{\perp}^{2}} f^{a b_{2} c} t^{c} t^{b_{1}} \\
& \times \frac{2 p_{+} q_{1-}}{\left[\left(k-q_{1}\right)^{2}+i 0\right]\left[\left(p+q_{2}\right)^{2}+i 0\right]} .
\end{aligned}
$$

The terms $D_{1}$ and $E_{1}$ summed with $A+B+C$ give

$$
\begin{aligned}
A & +B+C+D_{1}+E_{1}=-32 i(k l)^{2} \\
& \times\left(f^{b_{1} a c} t^{b_{2}} t^{c} \frac{L\left(p, q_{1}\right)}{\left(k^{\prime}+q_{2}\right)^{2}+i 0}+f^{b_{2} a c} t^{c} t^{b_{1}} \frac{L\left(p, q_{2}\right)}{\left(k-q_{1}\right)^{2}+i 0}\right),
\end{aligned}
$$

which corresponds to the diagrams of Fig. $4 A, B$ with normal Feynman propagators.

Interchanging $(1 \leftrightarrow 2)$ in $D_{2}$ and $E_{2}$ gives contributions $\tilde{D}_{2}$ and $\tilde{E}_{2}$. One finds

$$
\begin{aligned}
D_{2}+\tilde{E}_{2}= & 32(k l)^{2} \frac{\left(e, p+q_{1}\right)_{\perp}}{\left(p+q_{1}\right)_{\perp}^{2}} f^{a b_{1} c} f^{b_{2} c d} t^{d} \\
& \times \frac{p_{+}}{k_{+}} \frac{1}{\left(p+q_{1}\right)^{2}+i 0}
\end{aligned}
$$

and $\tilde{D}_{2}+E_{2}$ is obtained after interchange $(1 \leftrightarrow 2)$. Summing this with $F,(11)$ and $\tilde{F}$ obtained after $(1 \leftrightarrow 2)$ we get

$F+\tilde{D}_{2}+E_{2}=32(k l)^{2} f^{a b_{2} c} f^{b_{1} c d} t^{d} \frac{p_{+}}{k_{+}} \frac{B\left(p, q_{2}, q_{1}\right)}{\left(p+q_{2}\right)^{2}+i 0}$,

$\tilde{F}+D_{2}+\tilde{E}_{2}=32(k l)^{2} f^{a b_{1} c} f^{b_{2} c d} t^{d} \frac{p_{+}}{k_{+}} \frac{B\left(p, q_{1}, q_{2}\right)}{\left(p+q_{1}\right)^{2}+i 0}$.

These contributions correspond to the diagram in Fig. $4 C$ and the one with $(1 \leftrightarrow 2)$ with the Bartels vertex and normal Feynman propagator.

As a result the entire amplitude can be obtained from only diagrams in Fig. $4 A, B$, and $C$ with the standard Feynman propagators without the induced contribution in Fig. $4 D$. In this way, as before, the problem of singularities at $q_{1,2-}=0$ does not arise at all.

\section{Elastic scattering off three centers}

\subsection{Lowest order QCD}

In the axial gauge the amplitude for the elastic scattering off three centers in the lowest order is again trivial and reduces to the triple gluon exchange, Fig. $5 A$, since diagrams $5 B-D$ with both 3- and 4- gluon vertices give zero.

So the amplitude is

$$
A=64 i(k l)^{3} \frac{(321)}{\left[\left(k-q_{1}-q_{2}\right)^{2}+i 0\right]\left[\left(k-q_{1}\right)^{2}+i 0\right]}+P_{123} .
$$

where $P_{123}$ means adding contributions from the permutation of $(1,2,3)$. We recall that $(123)=t^{b_{1}} t^{b_{2}} t^{b_{3}}$. Denoting $q_{1-}, q_{2-}$, and $q_{3-}$ simply as 1,2 , and 3 respectively, we rewrite (25) as

$$
A=i \frac{64(k l)^{3}}{4 k_{+}^{2}} \frac{(321)}{[-(1+2)+i 0][-1+i 0]}+P_{123}
$$

For the following it is useful to split the propagators into the principal value and $\delta$-functional parts

$$
\begin{aligned}
& \frac{1}{[-(1+2)+i 0][-1+i 0]} \\
& =\left(-\frac{1}{1+2}-i \pi \delta(1+2)\right)\left(-\frac{1}{q_{1-}}-i \pi \delta(1)\right) \\
& =\frac{1}{(1+2) 1}+i \pi \frac{1}{q_{2-}}(\delta(1)-\delta(3))-\pi^{2} \delta(1) \delta(2)
\end{aligned}
$$

Here we used that in the Regge kinematics $1+2+3=0$.

In summing over permutations of $(1,2,3)$ it is convenient to combine terms with order (123) and (321), since

$2+3=-1,3=-(1+2), 3 \leftrightarrow 1$,

so that the term with $(123) \rightarrow(321)$ will have the same real part as (27) but the imaginary part with the opposite sign. Introducing

$$
(123)_{ \pm}=\frac{1}{2}((123) \pm(321))
$$

we find

$$
\begin{aligned}
A & =i \frac{64(k l)^{3}}{2 k_{+}^{2}}\left\{(321)_{+}\left(\frac{1}{(1+2) 1}-\pi^{2} \delta(1) \delta(2)\right)\right. \\
& +(213)_{+}\left(\frac{1}{(1+3) 3}-\pi^{2} \delta(1) \delta(3)\right) \\
& +(132)_{+}\left(\frac{1}{(2+3) 2}-\pi^{2} \delta(2) \delta(3)\right) \\
& +i \pi(321)_{-} \frac{1}{q_{2}}(\delta(1)-\delta(3))
\end{aligned}
$$




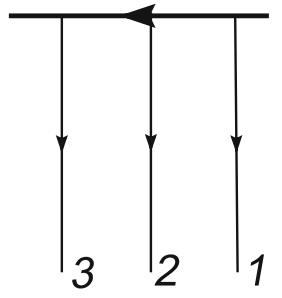

A

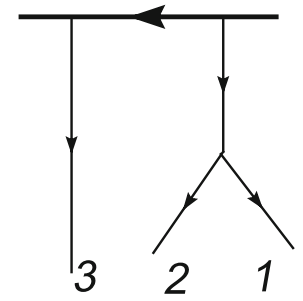

B

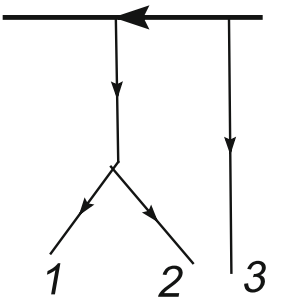

C

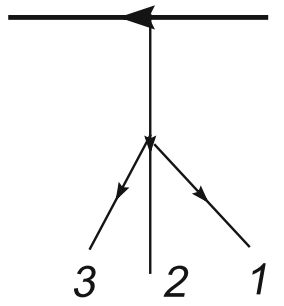

D

Fig. 5 Elastic scattering on three centers in the lowest order of the QCD

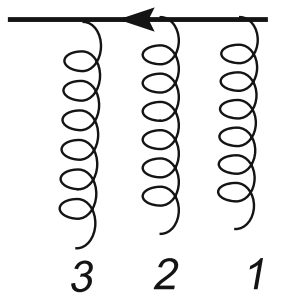

A

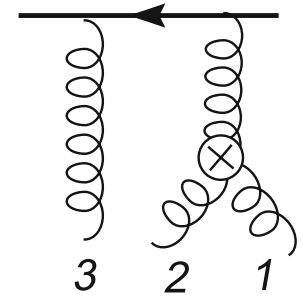

B

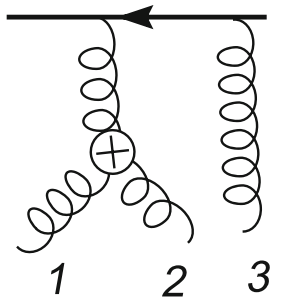

C

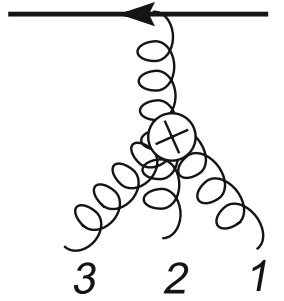

D

Fig. 6 Elastic scattering on three centers in the effective action approach

$$
\begin{aligned}
& +i \pi(213)_{-} \frac{1}{q_{1-}}(\delta(3)-\delta(2)) \\
& \left.+i \pi(132)_{-} \frac{1}{q_{3-}}(\delta(2)-\delta(1))\right\} .
\end{aligned}
$$

\subsection{Effective action result}

In the effective action approach, apart from the triple reggeon exchange we have additionally diagrams with induced 3reggeon and 4-reggeon vertices; see Fig. 6B-D.

In the triple gluon exchange we have to retain in the quark propagators only the $\delta$-functional terms. Their contribution will obviously reproduce terms with the product of two $\delta$ functions in (28).

Let us start with diagram $D$ in Fig. 6. The induced 4reggeon vertex is

$\Gamma_{R \rightarrow R R R}=2 i g^{2} \frac{q_{\perp}^{2}}{\left(q_{1}+q_{2}\right)-q_{1-}} \operatorname{Tr}\left(t^{a} t^{b_{3}} t^{b_{2}} t^{b_{1}}\right)+P_{123}$,

where $q=q_{1}+q_{2}+q_{3}$. We denote

$\operatorname{Tr}\left(t^{a} t^{b_{3}} t^{b_{2}} t^{b_{1}}\right) \equiv[a 321]$

and rewrite (29) as

$\Gamma_{R \rightarrow R R R}=2 i g^{2} \frac{q_{\perp}^{2}}{(1+2) 1}[a 321]+P_{123}$.

Combining terms with order (123) and (321) and assuming that the denominators do not vanish we get

$$
\begin{aligned}
\Gamma_{R \rightarrow R R R}= & 4 g^{2} q_{\perp}^{2}\left\{[a 321]_{+} \frac{1}{(1+2) 1}+[a 213]_{+}\right. \\
& \left.\times \frac{1}{(1+3) 3}+[a 132]_{+} \frac{1}{(3+2) 2}\right\}
\end{aligned}
$$

where $[a 132]_{+}=(1 / 2)([a 132]+[a 231])$.

Inserting this into the amplitude we get the additional factor $16 k_{+} l_{-}^{3} t^{a} / q_{\perp}^{2}$ with summation over $a$. Presenting $(123)=$ $C^{123}+C^{d 123} t^{d}$, multiplying, and taking traces we find

$[123]=N_{c} C^{123}=\frac{1}{4}\left(d^{123}+i f^{123}\right), \quad[a 123]=\frac{1}{2} C^{a 123}$.

Thus

$(123)=\frac{1}{4 N_{c}}\left(d^{123}+i f^{123}\right)+2 t^{a}[a 123]$,

from which we find

$$
\begin{aligned}
t^{a}[a 123] & =\frac{1}{2}(123)-\frac{1}{8 N_{c}}\left(d^{123}+i f^{123}\right), \quad t^{a}[a 123]_{+} \\
& =\frac{1}{2}(123)_{+}-\frac{1}{8 N_{c}} d^{123}
\end{aligned}
$$

So we get for diagram $D$ in Fig. 6

$$
\begin{aligned}
D= & 32 i k_{+} l_{-}^{3}\left\{(321)_{+} \frac{1}{(1+2) 1}+(213)_{+} \frac{1}{(1+3) 3}\right. \\
& \left.+(132)_{+} \frac{1}{(3+2) 2}\right\}+\Delta D
\end{aligned}
$$

where 


$$
\begin{aligned}
\Delta D & =32 i k_{+} l_{-}^{3} \frac{d^{123}}{8 N_{c}}\left\{\frac{1}{(1+2) 1}+\frac{1}{(1+3) 3}+\frac{1}{(3+2) 2}\right\} \\
& =-32 i k_{+} l_{-}^{3} \frac{d^{123}}{8 N_{c}}\left\{\frac{1}{1 \cdot 3}+\frac{1}{2 \cdot 3}+\frac{1}{1 \cdot 2}\right\}
\end{aligned}
$$

As mentioned in the Regge kinematics $1+2+3=0$. So if according to our rules we take all $q_{i-}, i=1,2,3$, different from zero then the r.h.s. of (34) vanishes. In the principal value prescription taken from the start

$$
\frac{1}{1 \cdot 3}+\frac{1}{2 \cdot 3}+\frac{1}{1 \cdot 2}=-\pi^{2} \delta(1) \delta(2) .
$$

So in this case the induced vertex contains a $\delta$-functional contribution. Our rule is equivalent to dropping it. So with our rule $\Delta D=0$ and comparing with (28) we conclude that the effective action correctly reproduces the part of the QCD amplitude with the product of two principal value poles.

Note that without our rule $\Delta D$ is different from zero and we get an additional contribution with the product $\delta(1) \delta(2)$, which spoils the agreement with the QCD result.

Now to the diagrams $B$ and $C$. Inserting the vertex $\Gamma_{R \rightarrow R R}$ into the amplitude we get the product

$i t^{b_{3}} t^{a} f^{a b_{1} b_{2}}=t^{b_{3}}\left[t^{b_{1}}, t^{b_{2}}\right]$

So we find

$$
\begin{aligned}
B & =16(k l) l_{-}^{2} \pi\left\{\delta(3) \frac{1}{q_{1-}}((312)-(321))\right. \\
& \left.+\delta(2) \frac{1}{q_{3-}}((231)-(213))+\delta(1) \frac{1}{q_{2-}}((123)-(132))\right\} .
\end{aligned}
$$

In the same way we find

$$
\begin{aligned}
C & =16(k l) l_{-}^{2} \pi\left\{\delta(3) \frac{1}{q_{1-}}((123)-(213))\right. \\
& \left.+\delta(2) \frac{1}{q_{3-}}((312)-(132))+\delta(1) \frac{1}{q_{2-}}((231)-(321))\right\} .
\end{aligned}
$$

In the sum

$$
\begin{aligned}
B+C= & 32(k l) l_{-}^{2} \pi\left\{(312)_{-}\left(\delta(3) \frac{1}{q_{1-}}+\delta(2) \frac{1}{q_{3-}}\right)\right. \\
& +(123)_{-}\left(\delta(3) \frac{1}{q_{1-}}+\delta(1) \frac{1}{q_{2-}}\right) \\
& \left.+(231)_{-}\left(\delta(2) \frac{1}{q_{3-}}+\delta(1) \frac{1}{q_{2-}}\right)\right\} .
\end{aligned}
$$

Taking into account that $1+2+3=0$ we can rewrite it as

$$
\begin{aligned}
B+C= & 32(k l) l_{-}^{2} \pi\left\{(312)_{-} \frac{1}{q_{1-}}(\delta(3)-\delta(2))\right. \\
& +(123)_{-} \frac{1}{q_{2}}(\delta(1)-\delta(3)) \\
& \left.+(231)_{-} \frac{1}{q_{3-}}(\delta(2)-\delta(1))\right\} .
\end{aligned}
$$

Comparing with (28) we conclude that we reproduce the QCD result with the pole singularities in the principal value prescription.

So in the end we have demonstrated that the principal value prescription for the effective action gives the correct amplitude for the elastic scattering on three centers provided one drops $\delta$-functional terms from the induced vertices and retains only those of them that come from the projectile quark propagators.

Note that as for the elastic scattering off two centers we can forget about the induced vertices and use only the triple reggeon exchange with full Feynman propagators for the projectile quark, thus completely avoiding discussion about pole singularities in $q_{i-}, i=1,2,3$.

\section{Gluon emission off three centers}

5.1 QCD results

\section{Three interactions of the projectile and the gluon emit- ted from it}

We have the four diagrams shown in Fig. 7A-D.

They all have a common factor $F_{1}$ :

$F_{1}=16 i \frac{(k l)^{3}}{8 k_{+}^{3}} \frac{k_{+}}{p_{+}}(e p)_{\perp}$

$\left(i^{7}\right.$ from the quark line, $(-i)^{3}$ from interactions, $(-i)$ for the amplitude and a minus from $(e k))$.

Now for the rest. To have a more symmetric notations we denote $p_{-} \equiv q_{4-}$ and $a \equiv b_{4}$. Then we find, neglecting the transverse parts

$$
\begin{aligned}
(7, A) & =\frac{(3214)}{(-4+i 0)(-(4+1)+i 0)(-(4+1+2)+i 0)} \\
& =\frac{(3214)}{(-4+i 0)(-(4+1)+i 0)(3+i 0)},
\end{aligned}
$$

where we have used $1+2+3+4=0$. Next

$$
\begin{aligned}
& (7 . B)=\frac{(3241)}{(-1+i 0)(-(4+1)+i 0)(3+i 0)}, \\
& (7 . C)=\frac{(3421)}{(-1+i 0)(-(1+2)+i 0)(3+i 0)}
\end{aligned}
$$




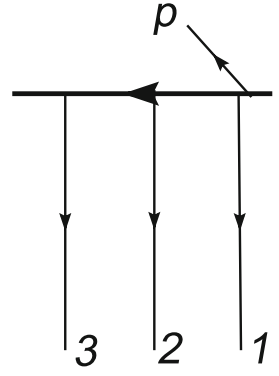

A
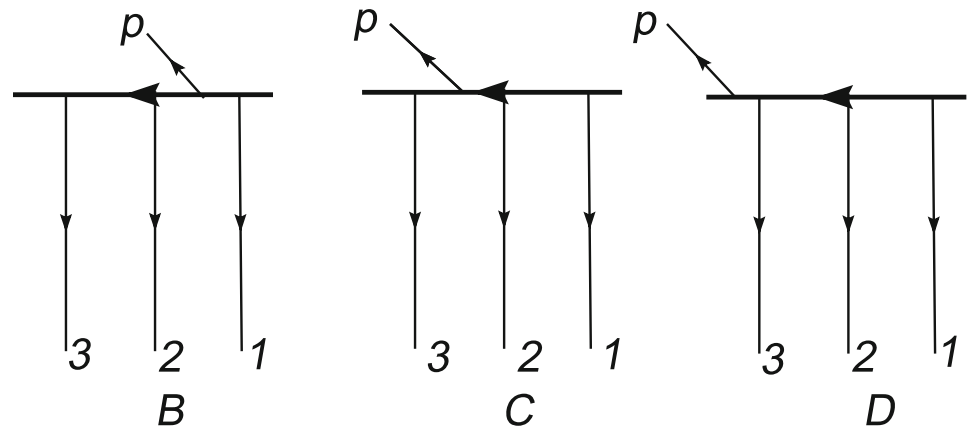

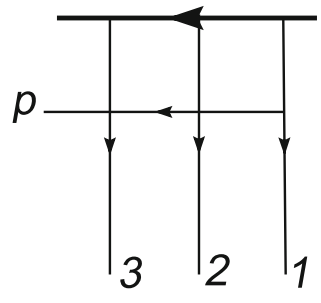

E

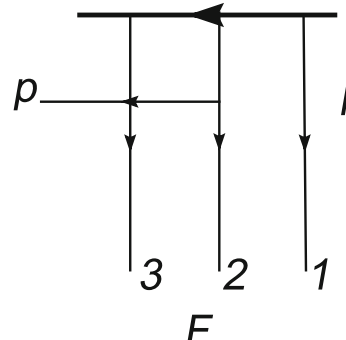

$F$

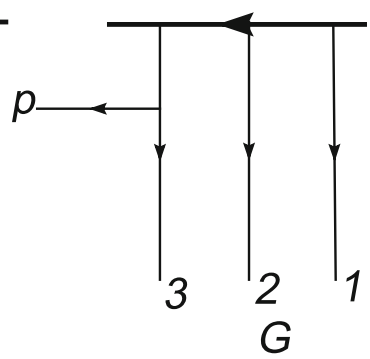

Fig. 7 Three interactions of the projectile with gluon emission

and finally

$\left(7 . D=\frac{(4321)}{(-1+i 0)(-(1+2)+i 0)(4+i 0)}\right.$.

We transform

$$
\begin{array}{r}
\frac{1}{(-1+i 0)(-(1+4)+i 0)}=\frac{1}{-4+i 0} \\
\times\left(\frac{1}{-q_{1-}+i 0}-\frac{1}{-\left(q_{1}+p\right)_{-}+i 0}\right)
\end{array}
$$

and correspondingly split (7.B) into two parts $(7 . B)=$ $(7 . B 1)+(7 . B 2)$, where

$$
\begin{aligned}
(7 . B 1) & =\frac{(3241)}{(-4+i 0)(-1+i 0)(3+i 0)}, \\
(7 . B 2) & =-\frac{(3241)}{(-4+i 0)(-(1+4)+i 0)(3+i 0)} .
\end{aligned}
$$

Similarly $(7 . C)=(7 . C 1)+(7 . C 2)$ with

$$
\begin{aligned}
(7 . C 1) & =\frac{(3421)}{(4+i 0)(-1+i 0)(3+i 0)}, \\
(7 . C 2) & =-\frac{(3421)}{(4+i 0)(-1+i 0)(3+4+i 0)} .
\end{aligned}
$$

Now we combine (7.A) with (7.B2), (7.D) with (7.C2) and (7.B1) with (7.C1) taking into account that $4=p_{-}$does not vanish. We get

$$
\begin{gathered}
(7 . A+B+C+D)=\frac{1}{p_{-}}\left\{\frac{i(32 d) f^{41 d}}{(-(1+4)+i 0)(3+i 0)}\right. \\
\left.+\frac{i(d 21) f^{43 d}}{(-1+i 0)(3+4+i 0)}+\frac{i(3 d 1) f^{42 d}}{(-1+i 0)(3+i 0)}\right\} .
\end{gathered}
$$

2. Three interactions of the projectile and the gluon emitted from one of the interactions

We have three diagrams shown in Fig. $7 E-G$. The common momentum factor is

$F_{2}=-16 \frac{(k l)^{3}}{4 k_{+}^{2}}$.

The rest gives

$$
\begin{aligned}
& (7 . E+F+G)=\frac{\left[e\left(p+q_{1}\right)\right]_{\perp}}{\left(p+q_{1}\right)^{2}+i 0} \frac{(32 d) f^{d 41}}{(-(1+4)+i 0)(3+i 0)} \\
& \quad+\frac{\left[e\left(p+q_{2}\right)\right]_{\perp}}{\left(p+q_{2}\right)^{2}+i 0} \frac{(3 d 1) f^{d 42}}{(-1+i 0)(3+i 0)} \\
& +\frac{\left[e\left(p+q_{3}\right)\right]_{\perp}}{\left(p+q_{3}\right)^{2}+i 0} \frac{(d 21) f^{d 43}}{(-1+i 0)(3+4+i 0)} .
\end{aligned}
$$

Summing with the contributions from the previous section we find the total contributions from three interactions of the projectile as

$$
\begin{aligned}
\text { (7) } & =4 \frac{(k l)^{3}}{k_{+}^{2}}\left\{\left(\frac{(e p)_{\perp}}{p_{\perp}^{2}}-\frac{\left[e\left(p+q_{1}\right)\right]_{\perp}}{\left(p+q_{1}\right)^{2}+i 0}\right)\right. \\
& \times \frac{(32 d) f^{41 d}}{(-(1+4)+i 0)(3+i 0)}+\left(\frac{(e p)_{\perp}}{p_{\perp}^{2}}-\frac{\left[e\left(p+q_{2}\right)\right]_{\perp}}{\left(p+q_{2}\right)^{2}+i 0}\right)
\end{aligned}
$$




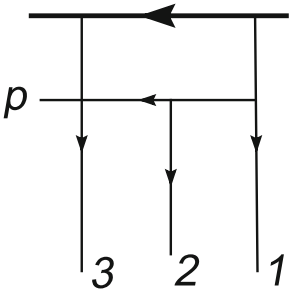

A

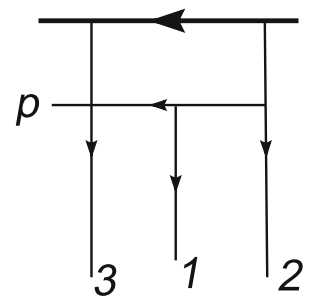

$B$

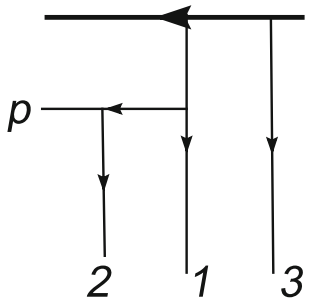

C

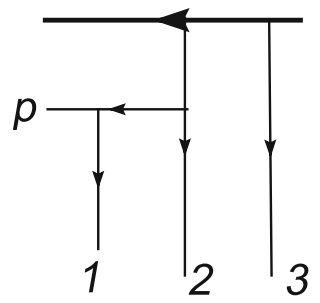

D

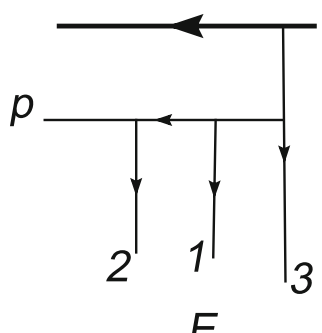

Fig. 8 Two interactions and one interaction of the projectile with gluon emission

$$
\begin{aligned}
& \times \frac{(3 d 1) f^{42 d}}{(-1+i 0)(3+i 0)}+\left(\frac{(e p)_{\perp}}{p_{\perp}^{2}}-\frac{\left[e\left(p+q_{3}\right)\right]_{\perp}}{\left(p+q_{3}\right)^{2}+i 0}\right) \\
& \left.\times \frac{(d 21) f^{43 d}}{(-1+i 0)(3+4+i 0)}\right\} .
\end{aligned}
$$

\section{Two interactions of the projectile}

Taking into account that diagrams with 3-gluon interaction with the target give zero, the total contribution from two interactions with the projectile reduces to four diagrams shown in Fig. 8A-D. Diagrams B and D are obtained from A and $\mathrm{C}$, respectively, by the interchange $1 \leftrightarrow 2$. So we only need to study A and C.

The common momentum factor is

$F_{3}=-16 i \frac{(k l)^{3} p_{+}}{2 k_{+}^{2}} \frac{\left[e\left(p+q_{1}+q_{2}\right)\right]_{\perp}}{\left(p+q_{1}+q_{2}\right)^{2}+i 0}$.

The rest gives

$$
\begin{aligned}
(8 . A)= & F_{3} \frac{(3 d) f^{d e 1} f^{e 42}}{\left(\left(p+q_{2}\right)^{2}+i 0\right)(3+i 0)}, \quad(8 . C)=F_{3} \\
& \times \frac{(d 3) f^{d e 1} f^{e 42}}{\left(\left(p+q_{2}\right)^{2}+i 0\right)(-3+i 0)} .
\end{aligned}
$$

\section{Single interaction of the projectile}

Taking into account that diagrams with 4-gluon interaction with the target give zero, the total contribution from the single interactions with the projectile reduces to the diagram shown in Fig. $8 E$ plus others which are obtained by permutations of the three gluons $1,2,3$.
The momentum factor is

$F_{4}=16 \frac{(k l)^{3} p_{+}^{2}}{k_{+}^{2}} \frac{\left[e\left(p+q_{1}+q_{2}+q_{3}\right)\right]_{\perp}}{\left(p+q_{1}+q_{2}+q_{3}\right)_{\perp}^{2}}$.

Note that $\left(p+q_{1}+q_{2}+q_{3}\right)_{-}=0$ so that in this case $\left(p+q_{1}+q_{2}+q_{3}\right)^{2}+i 0=\left(p+q_{1}+q_{2}+q_{3}\right)_{\perp}^{2}$. The rest gives the contribution from Fig. $8 D$ as

$$
(8 . E)=F_{4} \frac{t^{b} f^{b d 3} f^{d e 1} f^{e 42}}{\left(\left(p+q_{2}\right)^{2}+i 0\right)\left(\left(p+q_{2}+q_{1}\right)^{2}+i 0\right)}
$$

\subsection{Gluon emission off three centers in terms of the Lipatov and Bartels vertices}

To compare with the results of the effective action it is convenient to first express the QCD amplitude in terms of the Lipatov and Bartels vertices.

We start from the expression for diagrams in Fig. 7 obtained in the previous subsection. Since it is symmetric under permutations of gluons 1,2, and 3, we choose a different numeration of gluons in different diagrams, namely, 213 from the left in the first term, 132 in the second and 321 in the third one. Then instead of (41) we get

$$
\text { (7) } \begin{aligned}
= & 4 \frac{(k l)^{3}}{k_{+}^{2}}\left\{\left(\frac{(e p)_{\perp}}{p_{\perp}^{2}}-\frac{\left[e\left(p+q_{3}\right)\right]_{\perp}}{\left(p+q_{3}\right)^{2}+i 0}\right)\right. \\
& \times \frac{(21 d) f^{43 d}}{(-(3+4)+i 0)(2+i 0)}+\left(\frac{(e p)_{\perp}}{p_{\perp}^{2}}-\frac{\left[e\left(p+q_{3}\right)\right]_{\perp}}{\left(p+q_{3}\right)^{2}+i 0}\right)
\end{aligned}
$$




$$
\begin{aligned}
& \times \frac{(1 d 2) f^{43 d}}{(-2+i 0)(1+i 0)}+\left(\frac{(e p)_{\perp}}{p_{\perp}^{2}}-\frac{\left[e\left(p+q_{3}\right)\right]_{\perp}}{\left(p+q_{3}\right)^{2}+i 0}\right) \\
& \left.\times \frac{(d 21) f^{43 d}}{(-1+i 0)(3+4+i 0)}\right\} .
\end{aligned}
$$

We use the identity

$$
\frac{1}{\left(p+q_{3}\right)^{2}+i 0}=\frac{1}{\left(p+q_{3}\right)_{\perp}^{2}}-\frac{2 p_{+}\left(p+q_{3}\right)_{-}}{\left(p+q_{3}\right)_{\perp}^{2}\left(\left(p+q_{3}\right)^{2}+i 0\right)} \text {. }
$$

The first term in (44) converts the brackets in (43) into the Lipatov vertex and we get the first part of the amplitude:

$$
\begin{aligned}
A_{1}= & (7)_{1}=4 \frac{(k l)^{3}}{k_{+}^{2}} L\left(p, q_{3}\right)\left\{\frac{(21 d) f^{a 3 d}}{(-(3+4)+i 0)(2+i 0)}\right. \\
& \left.+\frac{(1 d 2) f^{a 3 d}}{(-2+i 0)(1+i 0)} \frac{(d 21) f^{a 3 d}}{(-1+i 0)(3+4+i 0)}\right\}+P_{123} .
\end{aligned}
$$

The second term in (44) cancels one of the denominators in each of the three terms in (43). The second term follows from

$\frac{2 p_{+}\left(p+q_{3}\right)_{-}}{\left(-q_{2-}+i 0\right)\left(q_{1-}+i 0\right)}=\frac{2 p_{+}}{q_{1-}+i 0}-\frac{2 p_{+}}{-q_{2-}+i 0}$,

since $\left(p+q_{3}\right)_{-}=-\left(q_{1}+q_{2}\right)_{-}$. We obtain the second part of (7):

$$
\begin{aligned}
(7)_{2}= & 4 \frac{(k l)^{3}}{k_{+}^{2}} f^{43 d} \cdot \frac{\left[e\left(p+q_{3}\right)\right]_{\perp}}{\left(p+q_{3}\right)_{\perp}^{2}} \frac{2 p_{+}}{\left(p+q_{3}\right)^{2}+i 0} \\
& \times\left\{-\frac{(21 d)}{2+i 0}+\frac{(1 d 2)}{1+i 0}-\frac{(1 d 2)}{-2+i 0}+\frac{(d 21)}{-1+i 0}\right\} .
\end{aligned}
$$

Now we interchange $1 \leftrightarrow 2$ in the first and third terms in (47) and change $d \rightarrow e$ after which it takes the form

$$
\begin{aligned}
\text { (7) } & =8 i \frac{(k l)^{3}}{k_{+}^{2}} \cdot p_{+} \frac{\left[e\left(p+q_{3}\right)\right]_{\perp}}{\left(p+q_{3}\right)_{\perp}^{2}} \\
\times & \left\{\frac{(1 d) f^{d e 2} f^{e 43}}{\left(\left(p+q_{3}\right)^{2}+i 0\right)(1+i 0)}+\frac{(d 1) f^{d e 2} f^{e 43}}{\left(\left(p+q_{3}\right)^{2}+i 0\right)(-1+i 0)}\right\} .
\end{aligned}
$$

Passing to the contribution in Fig. $8 A-D$ we choose the order of gluons from left to right (132) for (8.A) and (321) for (8.C). Then their sum can be rewritten as

$$
\begin{aligned}
(8 . A+C)= & -8 i \frac{(k l)^{3}}{k_{+}^{2}} \frac{\left[e\left(p+q_{3}+q_{2}\right)\right]_{\perp}}{\left(p+q_{3}+q_{2}\right)^{2}+i 0} \\
& \times\left(\frac{(1 d) f^{d e 2} f^{e 43} p_{+}}{\left.\left(p+q_{3}\right)^{2}+i 0\right)(1+i 0)}\right. \\
& \left.+\frac{(d 1) f^{d e 2} f^{e 43} p_{+}}{\left.\left(p+q_{3}\right)^{2}+i 0\right)(-1+i 0)}\right) .
\end{aligned}
$$

Putting into (49) the first term of the right-hand side of the identity

$$
\begin{aligned}
& \frac{1}{\left(p+q_{3}+q_{2}\right)^{2}+i 0}=\frac{1}{\left(p+q_{3}+q_{2}\right)_{\perp}^{2}} \\
& -\frac{2 p_{+}\left(p+q_{3}+q_{2}\right)_{-}}{\left(p+q_{3}+q_{2}\right)_{\perp}^{2}\left(\left(p+q_{3}+q_{2}\right)^{2}+i 0\right)}
\end{aligned}
$$

and summing with (48) we get the second term of the amplitude:

$$
\begin{aligned}
A_{2}= & 4 \frac{(k l)^{3}}{k_{+}^{2}} \cdot i \frac{2 p_{+} B\left(p, q_{3}, q_{2}\right)}{\left(p+q_{3}\right)^{2}+i 0} \\
& \times\left(\frac{(1 d) f^{d e 2} f^{e 43}}{1+i 0}+\frac{(d 1) f^{d e 2} f^{e 43}}{-1+i 0}\right)+P_{123} .
\end{aligned}
$$

Putting into (49) the second term of the right-hand side of (50) we find

$$
\begin{aligned}
-16 & \frac{(k l)^{3}}{k_{+}^{2}} \frac{\left[e\left(p+q_{3}+q_{2}\right)\right]_{\perp}}{\left(p+q_{3}+q_{2}\right)_{\perp}^{2}} \\
& \times \frac{t^{b} f^{b d 1} f^{d e 2} f^{e a 3} \cdot p_{+}^{2}}{\left(\left(p+q_{3}\right)^{2}+i 0\right)\left(\left(p+q_{3}+q_{2}\right)^{2}+i 0\right)} .
\end{aligned}
$$

Summing this with the contribution (42) from Fig. $8 E$ we find the third term of the amplitude

$$
\begin{aligned}
A_{3}= & -4 \frac{(k l)^{3}}{k_{+}^{2}} B\left(p, q_{3}+q_{2}, q_{1}\right) \\
& \times \frac{4 p_{+}^{2} \cdot t^{b} f^{b d b_{1}} f^{d e b_{2}} f^{e a b_{3}}}{\left(\left(p+q_{3}\right)^{2}+i 0\right)\left(\left(p+q_{3}+q_{2}\right)^{2}+i 0\right)}+P_{123} .
\end{aligned}
$$

As a result we presented the QCD amplitude as a sum of contributions corresponding to the transverse picture with vertices of Lipatov and Bartels with normal Feynman propagators both for gluons and quarks and illustrated in Fig. 9.

\subsection{The effective action result}

To find the contribution for the amplitude following application of the effective action we can use our results in [12] where the most complicated $\mathrm{R} \rightarrow \mathrm{RRRP}$ vertex was constructed under the assumption that neither of $q_{i-}, i=1,2,3$ vanishes. It contributes to the part of the production amplitude $A_{I}$ with a single interaction with the quark projectile. The contribution $A_{I}$ was found to be ${ }^{1}$

$$
A_{I}=g^{4} \gamma_{+} t^{d} f^{d 1 c} f^{c 2 d} f^{d 3 a}\left(W_{I}+Q_{I}+R_{I}\right)+P_{123}
$$

\footnotetext{
${ }^{1}$ Note that the results of [12] were rewritten here in correspondence with the normalization $q_{ \pm}=\left(q_{0} \pm q_{3}\right) / \sqrt{2}$ accepted in the present paper.
} 
Fig. 9 Gluon emission on three centers in the lowest order of the QCD presented in terms of Lipatov and Bartels vertices
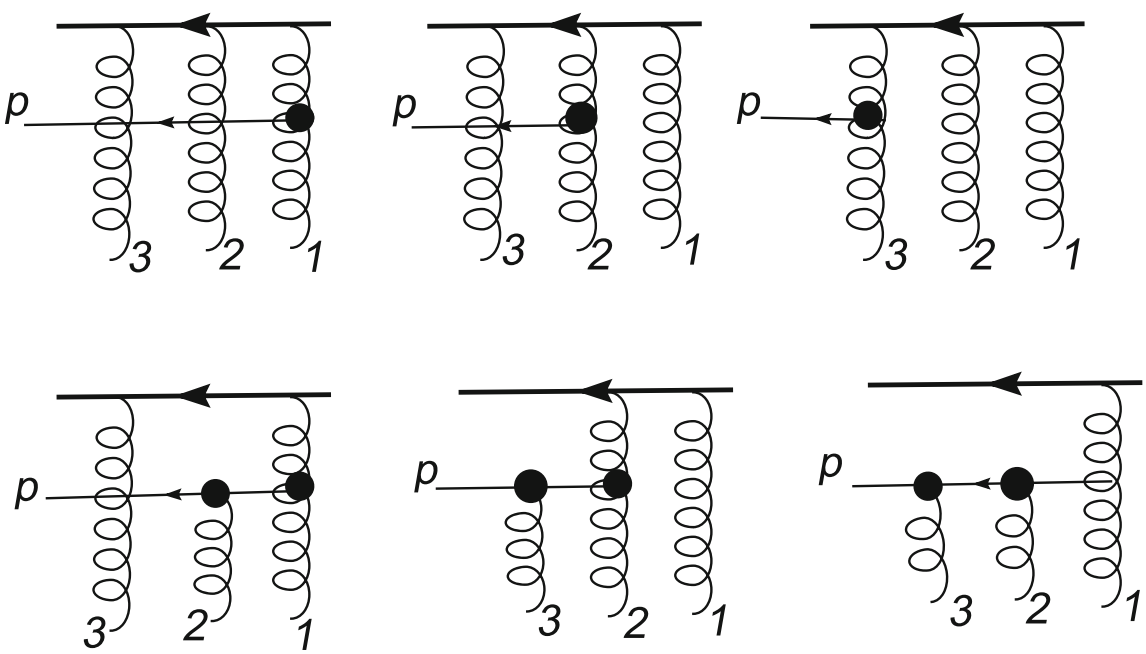

where

$W_{I}=\frac{2 q_{+}^{2} B\left(p, q_{3}+q_{2}, q_{1}\right)}{\left(\left(q-q_{1}\right)^{2}+i 0\right)\left(\left(q-q_{1}-q_{2}\right)^{2}+i 0\right)}$,

$Q_{I}=-\frac{q_{+} B\left(p, q_{3}, q_{2}\right)}{q_{1-}\left(\left(q-q_{1}-q_{2}\right)^{2}+i 0\right)}$,

$R_{I}=\frac{L\left(p, q_{3}\right)}{2 q_{1-}\left(q_{1-}+q_{2-}\right)}$.

Here $q=p+q_{1}+q_{2}+q_{3}$; it is assumed that $q_{1-},\left(q_{1-}+\right.$ $\left.q_{2-}\right) \neq 0$.

In the framework of effective action to this contribution one has to add the ones with the double and triple interactions of the projectile. In both cases only the $\delta$-functional parts of the quark propagators are to be retained. In this manner we find for the double interaction with the projectile

$$
\begin{aligned}
A_{I I} & =-i \pi g^{4} \gamma_{+} f^{d 3 a}\{((2 d 1)-(d 21)+(12 d)-(1 d 2)) \delta(1) \\
& \times\left[\frac{q_{+} B\left(p, q_{3}, q_{2}\right)}{\left(q-q_{1}-q_{2}\right)^{2}+i 0}-\frac{L\left(p, q_{3}\right)}{2 q_{2}-}\right] \\
& \left.+\delta(1+2) L\left(p, q_{3}\right)\left(\frac{(d 21)}{2 q_{1-}}-\frac{(21 d)}{2 q_{2}-}\right)\right\}+P_{123}
\end{aligned}
$$

and for the triple interaction with the projectile

$$
\begin{aligned}
A_{I I I}= & \frac{1}{2} g^{4} \gamma_{+} \pi^{2} \delta(1) \delta(2) f^{d 3 a}((d 21)+(1 d 2)+(21 d)) \\
& \times L\left(p, q_{3}\right)+P_{123} .
\end{aligned}
$$

Summing all contributions we first find the term coming from $W_{I}$ in (56) which exactly reproduces the QCD term $A_{3}$ (53). The part $Q_{I}$ summed with the term with $B\left(p, q_{3}, q_{2}\right)$ in $A_{I I}$ gives

$$
\begin{aligned}
& g^{4} \gamma_{+} f^{d b_{3} a} \frac{q_{+} B\left(p, q_{3}, q_{2}\right)}{\left(q-q_{1}-q_{2}\right)^{2}+i 0} \\
& \quad \times\left[((2 d 1)-(d 21))\left(-\frac{1}{q_{1-}}-i \pi \delta\left(q_{1-}\right)\right)\right. \\
& \left.\quad+((12 d)-(1 d 2))\left(\frac{1}{q_{1-}}-i \pi \delta\left(q_{1-}\right)\right)\right]+P_{123} .
\end{aligned}
$$

We observe that this expression will coincide with the contribution $A_{2}$ from the QCD provided we interpret the poles at $q_{1-}=0$ in the principal value sense.

The sum of all the rest terms can be presented as

$$
\begin{aligned}
& \frac{(2 d 1)_{+}-(d 21)_{+}}{1(1+2)}+\frac{(d 21)_{-}+(1 d 2)_{-}}{q_{2-}}(-i \pi) \delta(1) \\
& +\frac{(d 21)_{-}}{q_{1-}}(-i \pi) \delta(1+2)-\frac{1}{2}\left((d 21)_{+}+(1 d 2)_{+}+(21 d)_{+}\right) \\
& \times(-i \pi)^{2} \delta(1) \delta(2)+(1 \leftrightarrow 2)
\end{aligned}
$$

multiplied by the factor $g^{4} \gamma_{+} f^{d 3 a} L\left(p, q_{3}\right)$. Note that the first term comes from $R_{I}$ in the induced $R \rightarrow R R R P$ vertex and has been obtained under the assumption that $q_{1,2,3-}$, are different from zero, while the rest of the terms involve contributions just from their zero values, coming from the rescattering of the quark projectile.

In the sum with $1 \leftrightarrow 2$ in the first term there appears a contribution

$$
(2 d 1)_{+}\left(\frac{1}{1(1+2)}+\frac{1}{2(1+2)}\right) .
$$

With the principal value poles the bracket is

$$
\frac{1}{1(1+2)}+\frac{1}{2(1+2)}=\frac{1}{q_{1-}} \frac{1}{q_{2-}}+\pi^{2} \delta(1) \delta(2) .
$$

As we observe in this case the induced vertex again has a $\delta$-functional contribution, which we must drop, according to 
our rule. Then (61) becomes

$$
(2 d 1)+\frac{1}{q_{1}-} \frac{1}{q_{2-}}
$$

and one can combine all terms in (60) into

$$
\begin{aligned}
& \frac{1}{2}(d 21)\left(-\frac{1}{q_{1}} \frac{1}{1+2}+(-i \pi) \delta(1) \frac{1}{1+2}\right. \\
& \left.\quad+(-i \pi) \delta(1+2) \frac{1}{q_{1-}}-(-i \pi)^{2} \delta(1+2) \delta(1)\right) \\
& \quad+\frac{1}{2}(1 d 2)\left(\frac{1}{q_{1-}} \frac{1}{q_{2-}}+(-i \pi) \delta(1) \frac{1}{q_{2-}}\right. \\
& \left.\quad-(-i \pi) \delta(2) \frac{1}{q_{1-}}-(-i \pi)^{2} \delta(1) \delta(2)\right) \\
& \quad+\frac{1}{2}(21 d)\left(-\frac{1}{q_{2}-} \frac{1}{1+2}-(-i \pi) \delta(2) \frac{1}{1+2}\right. \\
& \left.\quad-(-i \pi) \delta(1+2) \frac{1}{q_{2-}}-(-i \pi)^{2} \delta(1+2) \delta(2)\right)+(1 \leftrightarrow 2) .
\end{aligned}
$$

With all poles at $q_{1-}=0, q_{2-}=0$, and $q_{1-}+q_{2-}=0$ taken in the principal value prescription (64) can be written as

$$
\begin{aligned}
- & \frac{1}{2}\left\{\frac{(d 21)}{(-(1+2)+i 0)(-1+i 0)}+\frac{(1 d 2)}{(1+i 0)(-2+i 0)}\right. \\
& \left.+\frac{(21 d)}{(2+i 0)(1+2+i 0)}\right\}+(1 \leftrightarrow 2) .
\end{aligned}
$$

Restoring the suppressed factor we see that this contribution exactly coincides with the part $A_{1}$ of the QCD contribution, given by (45). Thus, if we drop the $\delta$-functional term in (62), the effective action approach give the correct QCD amplitude for gluon emission on three centers.

Note that this is not the only place where $\delta$-functional terms appear in the induced vertex. In fact already the expression for the $\mathrm{R} \rightarrow \mathrm{RRRP}$ vertex in $A_{I}$ was obtained after dropping such terms. Calculations find $\delta$-functional terms in the induced $\mathrm{R} \rightarrow \mathrm{RRP}$ vertex,

$$
\begin{aligned}
& \Delta \Gamma_{R \rightarrow R R P}=-i g^{2} \pi^{2} \delta(1) \delta(2)\left(p+q_{1}+q_{2}\right)_{\perp}^{2} \\
& \quad \times \frac{(p e)_{\perp}}{2 p_{+}}[b 1 a 2+b 2 a 1]+P_{12},
\end{aligned}
$$

and in the induced $\mathrm{R} \rightarrow \mathrm{RRRP}$ vertex,

$$
\begin{aligned}
& \Delta \Gamma_{R \rightarrow R R R P}=-g^{3} \pi^{2} \delta(1) \delta(2) q_{\perp}^{2} \frac{(p e)_{\perp}}{p_{\perp}^{2}} \\
& \quad \times\left([b d 21+b 12 d] f^{d 3 a}+\frac{1}{2} f^{b 1 c} f^{c 2 d} f^{d 3 a}\right) .
\end{aligned}
$$

Collecting all of them we find that if they are retained the effective action result for the amplitude will differ from the
QCD by the extra term

$g^{4} \frac{1}{8 N_{c}} d^{2 d 1} f^{d 3 a} \gamma_{+} L\left(p, q_{3}\right) \pi^{2} \delta(1) \delta(2)+P_{123}$.

\section{Discussion}

Two main results have been obtained in this paper. First we have demonstrated that for collisions off two and three centers in the Regge kinematics high-energy amplitudes both with and without gluon emission can be presented in terms of the reggeon exchange with vertices of Lipatov and Bartels for gluon emission with the standard Feynman propagators. This greatly simplifies practical calculations of physical probabilities. For the specific kinematical region $p_{-}<<q_{1,2-}$ this result was already found earlier [4]. In this paper we have demonstrated that it valid in the general Regge kinematics. Note that we believe that this result has a more general validity and is true for any amplitudes. This can be trivially demonstrated for the simple BFKL chain. Discussion of more complicated examples can be found in [13].

Second we have formulated a rule which guarantees that the effective action approach gives results coinciding with the QCD. This rule has the complementarity property: crudely speaking, one has to retain only the $\delta$-functional terms in the projectile propagators and to drop such terms in the induced vertices, in which the poles at zero values of the transferred longitudinal momenta are to be taken in the Cauchy principal value sense. The more precise meaning of the latter procedure was explained in the Introduction.

Both results have been found in the lowest order of the perturbative approach in the spirit of the BFKL approach, in which higher orders are to be accompanied by evolution in rapidity. They also have been found only for collisions on two and three centers. We believe, however, that they preserve validity also for larger number of collision centers.

As mentioned in the Introduction a different approach was taken in $[5,6]$. M.Hentschinski proposed to project the induced vertices for transitions of a reggeon to three or more reggeons onto the maximally antisymmetric color states and add $\pm i \epsilon$ to the denominators which vanish in these vertices. He found that after this projection the dependence on the sign of $\epsilon$ vanishes and the vertices satisfy the desired properties of Bose symmetry and negative signature of the reggeon. It was checked that his recipe reproduced the QCD results for the gluon trajectory with one and two loops [14-16]. However, his prescription refers only to the induced vertices themselves and consequently to the amplitude with a single interaction of colliding particles and not to the one with several interactions as in our case. In the latter case the amplitude may contain color states different from those present in the induced vertex after M.Hentschinski's projection. We calculated the pro- 
duction amplitude off three centers with his prescription for the vertices and only the $\delta$-functional parts of the rescattering projectile propagators retained, as dictated by the Regge kinematics. As a result we found an extra (maximally symmetric) term compared to the QCD,

$$
\begin{aligned}
& \frac{1}{6} g^{4} \pi^{2} \gamma_{+} L\left(p, q_{3}\right) \delta(1) \delta(2) f^{d 3 a} \\
& \quad \times\left([12 d]_{+}+[2 d 1]_{+}+[d 12]_{+}\right)+P_{123},
\end{aligned}
$$

so that the prescription does not work.

Of course this result is valid only within our procedure to treat multiple Regge exchanges (as in Fig. 6A), which we consider well founded [4]. We admit that with a different procedure applied to Fig. $6 A$ the prescription of $[5,6]$ may work. However, we see that one has first to propose an alternative procedure for Fig. $6 \mathrm{~A}$ compatible with the Regge kinematics.

Acknowledgments The authors are most thankful to J. Bartels and G.P. Vacca for several helpful and constructive discussions. The authors acknowledge Saint-Petersburg State University for a research grant 11.38.31.2011. This work has been also supported by the RFFI grant 1202-00356-a. M.A.B. is indebted to the Second Institute of Theoretical Physics at Hamburg university for hospitality.

Open Access This article is distributed under the terms of the Creative Commons Attribution License which permits any use, distribution, and reproduction in any medium, provided the original author(s) and the source are credited.

Funded by $\mathrm{SCOAP}^{3}$ / License Version CC BY 4.0.

\section{References}

1. L.N. Lipatov, Nucl. Phys. B 452, 369 (1995)

2. L.N. Lipatov, Phys. Rep. 286, 131 (1997)

3. M.A. Braun, M.I. Vyazovsky, Eur. Phys. J. C 51, 103 (2007)

4. M.A. Braun, L.N. Lipatov, MYu. Salykin, M.I. Vyazovsky, Eur. Phys. J. C 71, 1639 (2011)

5. M. Hentschinski, PhD. Thesis. arXiv:0908.2576

6. M. Hentschinski, Nucl. Phys. B 859, 129 (2012). arXiv:1112.4509

7. L.N. Lipatov, Sov. J. Nucl. Phys. 23, 338 (1976)

8. E.A. Kuraev, L.N. Lipatov, V.S. Fadin, Sov. Phys. JETP 45, 199 (1977)

9. I.I. Balitsky, L.N. Lipatov, Sov. J. Nucl. Phys. 28, 822 (1978)

10. J. Bartels, Nucl. Phys. B 175, 365 (1980)

11. M.A. Braun, S.S. Pozdnyakov, MYu. Salykin, M.I. Vyazovsky, Eur. Phys. J. C 73, 2572 (2013)

12. M.A. Braun, S.S. Pozdnyakov, MYu. Salykin, M.I. Vyazovsky, Eur. Phys. J. C 72, 2223 (2012)

13. M.A. Braun, Eur. Phys. J. C 73, 2511 (2013)

14. M. Hentschinski, A. Sabio Vera, Phys. Rev. D 85056006 (2012). arxiv: 1110.6741

15. G. Chachamis, M. Hentschinski, J.D. Madrigal Martínez, A. Sabio Vera, Nucl. Phys. B 861133 (2012). arXiv:1202.0649

16. G. Chachamis, M. Hentschinski, J.D. Madrigal Martínez, A. Sabio Vera, Nucl. Phys. B 876453 (2013). arXiv:1307.2569 